Netzwerk gegen chronischen Schmerz

\section{Forschung auf Europakurs}

I WIRKSAME THERAPIESTRATEGIEN Dem Deutschen Forschungsverbund Neuropathischer Schmerz (DFNS) ist es gelungen, sich als Partner im europäischen Forschungsnetzwerk "Europain" zu etablieren. Europain wird von der Innovative Medicines Initiative (IMI) gefördert und widmet sich dem Ziel, die Therapie chronischer Schmerzen nachhaltig zu verbessern.

D as vom Bundesministerium für Bildung und Forschung (BMBF) geförderte deutsche Netzwerk DFNS forscht seit 2002 in den Bereichen Pathophysiologie, Prävention und Therapie neuropathischer Schmerzen. Die Vernetzung aller wichtigen Kräfte und Fachrichtungen in Deutschland stellt einen der Meilensteine des Verbundes dar.

Europäische Förderung gesichert Dem Verbund ist es nun gelungen, auch im europäischen Forschungsnetzwerk Europain Fuß zu fassen. Europain wird von der Innovative Medicines Initiative (IMI) gefördert, einer öffentlich-privaten Partnerschaft zwischen der Europäischen Union, vertreten durch die Europäische Kommission, und der pharmazeutischen Industrie, vertreten durch den Europäischen Dachverband EFPIA (European Federation of Pharmaceutical Industries and Associations). Europain wird über die nächsten fünf Jahre mit insgesamt 18,5 Millionen EUR unterstützt.

\section{Gebündelte Expertise}

Die Projekte von Europain fokussieren auf chronische Schmerzerkrankungen, darunter auch neuropathische Schmerzen. Zu den Zielen gehört, das Verständnis der dem chronischen Schmerz zugrunde liegenden Mechanismen maßgeblich zu erweitern, die Entwicklung neuartiger Pharmakotherapien zu erleichtern und verlässliche Biomarker für Schmerz zu entwickeln. Letztlich soll die Lebensqualität chronischer Schmerzpatienten nachhaltig verbessert werden.

Die Kooperation umfasst insgesamt 20 akademische und industrielle Partner aus ganz Europa, darunter Forscher zwölf renommierter Universitäten: King's College London (akademische Leitung), University College London, Imperial College London, University of Oxford, ChristianAlbrechts-Universität zu Kiel, Medizinische Fakultät Mannheim der Universität Heidelberg, Technische Universität München, Goethe Universität Frankfurt, Berufsgenossenschaftliches Universitätsklinikum Bergmannsheil GmbH/Ruhr-Universität Bochum, University Hospitals of Aarhus, Rigshospitalet Copenhagen, University of Southern Denmark und SME Neuroscience Technologies in Barcelona. Zum Netzwerk gehören zudem drei etablierte europäische Konsortien, die auf Schmerzforschung spezialisiert sind: das London Pain Consortium, das Danish Pain Research Centre sowie der DFNS. In die Kooperation fließen auch Forschungsressourcen und die Expertise europäischer pharmazeutischer Unternehmen ein, die im analgetischen Bereich tätig sind, darunter: AstraZeneca (Koordinator), Boehringer Ingelheim, Eli Lilly, Esteve, Pfizer, SanofiAventis und UCB Pharma.

Dazu Prof. Thomas Tölle, Leiter der DFNS-Geschäftsstelle an der TU München, und gemeinsam mit Prof. Ralf Baron, Universität zu Kiel, Sprecher des DFNS: „Europain bietet uns die hervorragende Chance, unsere Expertise mit der unserer europäischen Kollegen zu bündeln. Mit der Ausdehnung unseres Netzwerkes auf den europäischen Raum sind wir auf dem besten Wege, ein Team aus weltweit kooperierenden experimentellen und $\mathrm{kli}$ nischen Schmerzforschern zu bilden."

\section{DFNS auf Europakurs}

Europain besteht aus verschiedenen Forschungsprojekten, die das wissenschaftliche und technische Know-how der beteiligten Partner optimal vernetzen, ergänzen und Synergien nutzen können. „Der DFNS kann auf seine mit über 2000 Patienten weltweit größte Datenbank zu neuropathischem Schmerz zugreifen und damit wichtige Referenzdaten in die Kooperation einbringen", so Prof. Christoph Maier, Universität Bochum, dessen Forschungsteam die Datenbank zentral verwaltet. Die Datenbank beinhaltet genaue quantitative Veränderungen der Hautsensibilität sowie psychologische Hintergründe von Patienten mit unterschiedlichen neuropathischen Schmerzbildern. Zusätzlich verfügt der Verbund über Biomarker in Form einer DNA-Bank, die zentral an der TU München verwaltet wird.

Zentraler Bestandteil des deutschen Forschungsprojekts ist auch das vom DFNS standardisierte Verfahren der Quantitativ Sensorischen Testung (QST). Die QST erlaubt eine genaue Analyse der Schmerzsymptomatik und gilt damit als Indikator für die zugrunde liegenden biologischen Mechanismen. Dazu Prof. RolfDetlef Treede, Universität Heidelberg: "Wir haben nun die Möglichkeit, das standardisierte QST-Protokoll des DFNS auf einer breiten Ebene in Europa zu etablieren und unsere Qualitätsrichtlinien mit einzubringen."

Abschließend Baron: "Die europäischen Projektpartner ergänzen unser Profil in optimaler Weise. Damit rücken wir unserem ultimativem Ziel - die medizinische Versorgung chronischer Schmerzpatienten grundlegend zu verbessern bedeutend näher."

Redaktion: Vedrana Romanovic, Pressestelle des DFNS (www. neuropathischer-schmerz.de) 